\title{
Oral Rehydration or Intravenous Drip? - A Volume Kinetic Study of Glucose Solutions to Young and Old
}

\section{Fredrik Sjöstrand ${ }^{1,2 *}$, Christer H Svensen ${ }^{3}$ and Peter M Rodhe}

${ }^{1}$ Department of Clinical Science and Education, Karolinska Institutet, Section of Emergency Medicine, Södersjukhuset, Stockholm, Sweden ${ }^{2}$ Head of Research and Education, Nacka Geriatric Clinic, Capio Geriatrik, Nacka, Sweden

${ }^{3}$ Senior lecturer, Director for Doctoral Education, Department of Clinical Science and Education, Karolinska Institutet, Section of Anesthesiology and Intensive Care, Södersjukhuset, Stockholm, Sweden

\begin{abstract}
Background: The aim of this study was to use a volume kinetic model to compare oral versus intravenous fluid administration of glucose solutions in young and elderly healthy volunteers. We investigated differences in the absorption, distribution and elimination of the administered fluid.

Methods: The study had a randomized, crossover design and all experiments were performed when subjects had been fasting overnight indicating a state of dehydration. Twenty-four healthy volunteers received an isotonic $2.5 \%$ glucose solution, $7 \mathrm{ml} / \mathrm{kg}$ during 15 minutes, both orally and intravenously at separate occasions. Repetitive blood tests over 120 minutes showed changes in body fluid volumes using volume kinetic principles.

Results: There were significant different lag times of absorption between oral and intravenous administration of fluid. In the young, a plasma volume decrease was shown in the beginning of the oral rehydration experiments but they retained fluid at the end of the experiment. The elderly had a consistent slow elimination of glucose and retained water after oral rehydration. The two administration routes showed different patterns for the elderly's fluid volume expansion with steeper slopes for intravenous rehydration.
\end{abstract}

Conclusions: It was possible to model the absorption, distribution and elimination of glucose fluids given both orally and intravenously in both age groups.

Keywords: Hypoaldosteronism; Glucose; Oral rehydration;

\section{Introduction}

Dehydration is a significant problem for the elderly population since the body composition of water changes during aging towards a drier state. Elderly subjects have a tendency to drink too little during daytime. This fact in combination with slight hypoaldosteronism, a reduced sensation of thirst, an impaired ability to concentrate urine and, in many cases, forgetfulness to drink can lead to significant dehydration and a cumbersome situation for both patients and caregivers. The consequences of dehydration are several and sometimes severe; conditions such as vertigo and imbalance leading to falls, urinary- and respiratory infections with or without septicaemia, delirium, renal failure and increased risk for medication toxicity. Elderly with many co-morbidities are at an increased risk for repeated hospitalizations [1-3] and dehydration has been associated with an increased mortality among hospitalized older adults [4].

It is often difficult to support elderly patients to drink sufficient amounts of fluids [5]. In today's health care, with decreasing hospital stays, oral rehydration is still the preferred choice while intravenous rehydration is though often used to more rapidly reach a normohydrated state. However, there is a lack of studies comparing rehydration models in not only the elderly but also in younger individuals.

The aim of this study was to compare two fluid administration routes, oral and intravenous administration, between a young and an elderly group of healthy volunteers. Primary outcomes were differences in the absorption, distribution and elimination of the administered fluid and glucose, respectively. These parameters were estimated by using a volume kinetic model [6-8].

As glucose often is added to water in the daily care of patients, and as the glucose molecules significantly affect the distribution and the elimination of the body fluid, there was a clear need to also analyze the kinetics of the glucose given to both age groups and types of administration. It is suggested in the literature that elderly have a $50 \%$ lower elimination rate of glucose [9] and this could have an effect also on the fluid balance.

The plasma volume expansion curves were of interest. Our hypothesis was that there should be a lag time in absorption when the fluid was given orally compared to intravenously. We anticipated that this lag time could be estimated with a volume kinetic model. Furthermore, we also anticipated that older subjects would have a slower elimination of both the glucose and the fluid given.

\section{Methods}

The study was designed as a prospective crossover study and was approved by the Ethical Review Board in the Stockholm County (Dno 2008/385-31/3). It was funded by the Stockholm County (PickUpFunding).

The experiments were performed at the Geriatric Department,

*Corresponding author: Fredrik Sjöstrand, Department of Clinical Science and Education, Karolinska Institutet, Section of Emergency Medicine, Södersjukhuset, Stockholm, Sweden, Tel: 46852480000; E-mail: fredrik.sjostrand@ki.se

Received September 24, 2014; Accepted October 15, 2014; Published November 20, 2014

Citation: Sjöstrand F, Svensen CH, Rodhe PM (2014) Oral Rehydration or Intravenous Drip?-A Volume Kinetic Study of Glucose Solutions to Young and Old. J Appl Computat Math 3: 190. doi:10.4172/2168-9679.1000190

Copyright: @ 2014 Sjöstrand F, et al. This is an open-access article distributed under the terms of the Creative Commons Attribution License, which permits unrestricted use, distribution, and reproduction in any medium, provided the original author and source are credited. 
Nacka Hospital, Sweden and at the Clinical Research Center at Karolinska Institutet, Section of Anesthesiology and Intensive Care, Södersjukhuset, Stockholm, Sweden.

Twenty-four volunteers were included in a young and an elderly group. They were recruited by responding to advertisements in the local newspaper, and gave their written consent to participate in the study after a medical examination including clinical assessment and a set of standard laboratory blood tests. All volunteers were required to be healthy. However, health in the very old age group is always a relative concept, so we accepted the diagnoses hypertension and hypothyroidism while cardiac diseases had to be restricted to New York Heart Association (NYHA) class I-II. Pregnancy, diabetes, diuretics and angiotensin converting enzyme inhibitors (ACEI) were conditions and treatments seen in those four individuals that were excluded from the study.

In the young group, twelve subjects aged 19-25 years (median 23) were recruited. Ten women with the median bodyweight of $64.5(60.6$ $-70.4) \mathrm{kg}$ and two men that weighed 87.2 and $71.8 \mathrm{~kg}$, respectively, were included.

In the elderly group, twelve subjects aged $74-88$ years (median 82 ) were recruited. Six women with the median bodyweight 66.1 (61.073.9) $\mathrm{kg}$ and six men with median bodyweight 83.6 (75.0-90.8) $\mathrm{kg}$ were included.

For both groups, no food or fluid intake was allowed after 10 PM the day before the experiment. On arrival to the research area, the volunteers were weighed after they had emptied the bladder. The urinary bladder was scanned at 0,15 and $120 \mathrm{~min}$ by an ultrasonic scanner BVI-3000 (Bladderscan", Allytec, Stockholm, Sweden). The volunteer was allowed to void during the experiment. The amount of voided urine was estimated. At the end of the experiment the patients were once again weighed before and after voiding.

Intravenous cannulas were placed in antecubital veins on each side. One cannula was used for blood sampling and the other for fluid infusion (for the intravenous experiment).

On separate occasions, the subjects in both groups were given a crystalloid, $25 \mathrm{mg} / \mathrm{ml}$ glucose solution, (Rehydrex ${ }^{\oplus}$ with ionic content $\mathrm{Na}^{+} 70 \mathrm{mmol}, \mathrm{Cl}^{-} 45 \mathrm{mmol}$, Ac $25 \mathrm{mmol}$ per $1000 \mathrm{ml}$ ) either orally (ORAL) or intravenously (IV) in a crossover design with at least a two week free interval between the experiments.

The subjects got $7 \mathrm{ml} / \mathrm{kg}$ of the crystalloid solution during 15 minutes on both occasions. During the ORAL experiment, the fluid was administered in fifteen cups and each cup represented $1 / 15$ the total amount. In the IV group, the administration of fluid was administered by the aid of an infusion pump (Fluido ${ }^{\circ}$, Amersfoort, Netherlands). Blood samples for hemoglobin and glucose were taken at time points $0,10,15,20,30,45,60,75,90,120$ minutes. Hemoglobin and hematocrit were measured by fluorescent flow cytometry (XE-5000, SYSMEX, Stockholm, Sweden). The plasma glucose concentration was measured in single samples with the GLU Gluco-quant reagent (Roche Diagnostic Inc., Mannheim, Germany) on a Hitachi 917 (Hitachi Co., Naka, Japan).

$70 \mathrm{ml}$ of blood were drawn from each subject during each experiment. The blood volume lost to sampling was replaced by the infusion of an equivalent amount of sodium chloride. During the experiment, the blood pressure was monitored by a non-invasive digital blood pressure monitor (Omron ${ }^{\circledR}$, Kyoto, Japan) at time points 0,15 and $120 \mathrm{~min}$.

\section{Mathematical Models}

\section{Glucose kinetics}

The one-compartment model of glucose kinetics, disregarding insulin and C-peptide kinetics, could be modelled by [6]:

$$
\frac{d C_{g}}{d t}=\frac{k_{i e x}+k_{i e n}-C L_{g} \cdot C_{g}}{V_{d}}, C_{g}(\mathrm{t}=0)=C_{g, 0}
$$

$C_{g}$ is the glucose concentration $(\mathrm{mmol} / \mathrm{ml})$.

$k_{i \text { ex }}$ and $k_{i \text { en }}$ are the rates of the exogenously administered glucose molecules and the endogenous production by the liver, respectively. The unit for these rates is $\mathrm{mmol} / \mathrm{ml} . C L_{g}$ is the glucose clearance (ml/ $\mathrm{min}$ ) and $V_{d}$ the volume of distribution for glucose ( $\mathrm{ml}$ ).

Moreover, due to the presence of exogenous glucose, the endogenous glucose production will be suppressed and the insulin levels will increase due to increased glucose concentration. Insulin and c-peptide data [10] were though not sampled. During some time after the glucose load, the previous steady-state, or basal level, $C_{g, 0}$ will be perturbed. The reason for introducing a baseline shift is because of the length of the experiment, which only captures data for the shortterm elimination of glucose. We modeled this baseline shift by a linear function $S_{g}$ depending on the scalar $S_{g, 0}$

$$
s_{g}(t)=\left\{\begin{array}{l}
s_{g, 0} \cdot \frac{t}{T}, t \leq T \\
s_{g, 0}, t>T
\end{array}\right.
$$

\section{Fluid kinetics}

The kinetics of the intravenously administrated isotonic 2.5\% glucose fluid, have previously been investigated by Sjöstrand et al. [11] (Figure 1).

$$
\frac{d v_{1}}{d t}=k_{i}-\frac{d U}{d t} \text { (one-compartment model) }
$$

where $k_{i}$ is the rate of infusion $(\mathrm{ml} / \mathrm{min}) . v_{1}$ is the volume of the central space (ml), and $V_{1}=v_{1}(0)$. $\mathrm{U}$ is the accumulated urine output (Figure 1).

For each mmol of glucose that enters the cellular fluid space, a specific amount of water $(\mathrm{ml})$, must accompany the glucose molecule in order to maintain the osmotic balance [11]. This will result in a

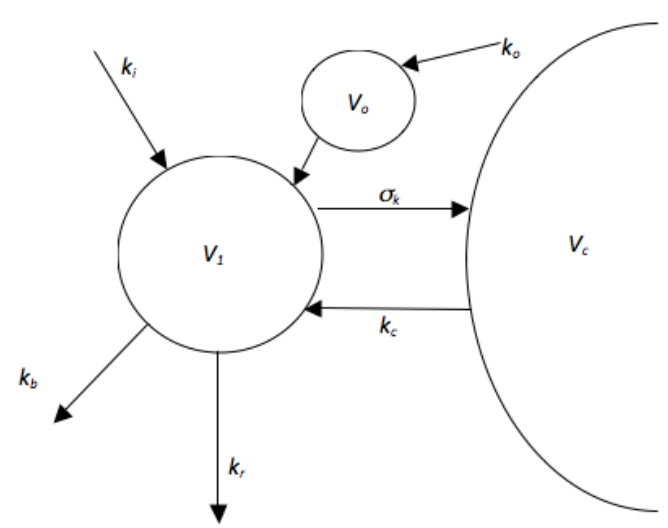

Figure 1: The two compartment fluid kinetic model. 
Citation: Sjöstrand F, Svensen CH, Rodhe PM (2014) Oral Rehydration or Intravenous Drip?-A Volume Kinetic Study of Glucose Solutions to Young and Old. J Appl Computat Math 3: 190. doi:10.4172/2168-9679.1000190

Page 3 of 6

temporary swelling of the cell and the excess water will eventually be transported out from the cell. For this model, the gradient of volume changes in the cellular fluid space, were modeled by:

$$
\frac{d v_{c}}{d t}=\sigma \cdot C L_{g} \cdot\left(C_{g}-C_{g, 0}\right)-k_{c} \frac{v_{c}-V_{c}}{V_{c}}
$$

Where $v_{c}$ is the volume of the cellular fluid space $(\mathrm{ml})$ and $k_{c}$ the parameter describing in what rate any excess water in ICF is transported out from $v_{c}$. Hence, the system of differential equations will appear as:

$$
\begin{aligned}
& \frac{d v_{1}}{d t}=k_{i}-\frac{d U}{d t}-\sigma \cdot C L\left(C_{g}-C_{g, 0}\right)+k_{c} \frac{v_{c}-V_{c}}{V_{c}} \\
& \frac{d v_{c}}{d t}=\sigma \cdot C L_{g}\left(C_{g}-C_{g, 0}\right)-k_{c} \frac{v_{c}-V_{c}}{V_{c}}
\end{aligned}
$$

Furthermore, during per oral fluid administration, a first-pass compartment $V_{\mathrm{o}}(\mathrm{ml})$ is added to the differential equations (Figure 1).

The urine output was modeled by an exponential function [12]:

$$
\frac{d U}{d t}=k_{b} \cdot e^{k_{c l} \cdot d i v_{1}}
$$

Where $\operatorname{dil} v_{1}=\frac{v_{1}-V_{1}}{V_{1}}, k_{e l}$ is the rate of elimination $(\mathrm{ml} / \mathrm{min})$ and $k_{b}$ is the basal elimination $(\mathrm{ml} / \mathrm{min})$.

\section{Bioavailibility}

The bioavailability was also estimated for the glucose effect and the volume effect respectively by:

$$
F=\frac{[A U C]_{p o}}{[A U C]_{i v}}
$$

in order to compare the routes per-oral and intravenously respectively.

\section{Half-life}

The half-life of glucose was estimated after computing $V_{d}$ and $C L$ by:

$$
t_{1 / 2}=\frac{V_{d}}{C L} \times \ln 2
$$

\section{Computation}

First, the glucose kinetic equations were solved by an ordinary differential equation solver (ode45, Matlab) while a minimization routine estimated the parameters (fminsearch, MatLab) (Table 1).

\section{Statistics}

Data are presented as medians and ranges for the $25^{\text {th }}$ and $75^{\text {th }}$ percentiles. ANOVA and nonparametric tests where appropriate. Test of homogeneity of variances was Levenes.

\section{Results}

The mean curves of glucose concentration and dilution of plasma volume are plotted in Figure 2A and 2B. Both parameters responded significantly to the administrated glucose solution.

The elderly eliminated glucose slower than the younger subjects, which was indicated by a sharper increase of the plasma glucose slope (Figure 2 and Table 2). At the end of the elderly's IV experiments, the plasma volumes returned to baseline but first showing a dip in plasma volume about 1 hour after the infusion had stopped. After 10 and

\begin{tabular}{|c|c|c|c|c|c|}
\hline & $C L$ & $V_{d}$ & $T_{0}$ & $t_{1 / 2}$ & $S_{g, 0}$ \\
\hline & $\mathrm{ml} / \mathrm{min}$ & $\mathrm{ml}^{*} 10^{-3} \S$ & $\min ^{*}$ & $\min$ & (\%) $\S^{\star *}$ \\
\hline PO-young & $950(710-1135)$ & $7.8(4.9-12.5)$ & $17.0(7.9-24.9)$ & $3.8(3.1-11.9)$ & $93.2(89.4-97.2)$ \\
\hline IV-young & $480(360-597)$ & $12.2(10.6-14.6)$ & - & $16.1(14.8-23.0)$ & $87.9(85.0-95.8)$ \\
\hline PO-elderly & $411(231-501) \S$ & $18.6(15.2-20.4)$ & $18.0(12.9-21.8)$ & $30.5(22.3-55.9)$ & $80.2(58.7-86.6)$ \\
\hline IV-elderly & $288(216-347) \S$ & $14.9(11.4-17.8)$ & - & $41.6(23.8-54.6)$ & $78.1(60.9-92.3)$ \\
\hline
\end{tabular}
15 minutes of PO intake of the isotonic glucose solution, the young subjects had a negative plasma dilution.

\begin{tabular}{|c|c|c|c|}
\hline Glucose kinetic parameters & Unit & Volume effect parameters & \multicolumn{1}{c|}{$\boldsymbol{k}_{\text {el }}$} \\
\hline $\boldsymbol{C L}$ & $\mathrm{ml} / \mathrm{min}$ & $\boldsymbol{k}_{\mathrm{b}}$ & $\mathrm{ml} / \mathrm{min}$ \\
\hline $\boldsymbol{V}_{\boldsymbol{d}}$ & $\mathrm{ml}$ & $\boldsymbol{T}_{\boldsymbol{v}}$ & $\mathrm{ml} / \mathrm{min}$ \\
\hline $\boldsymbol{T}_{0}$ & $\mathrm{~min}$ & $\boldsymbol{V}_{1}$ & $\mathrm{~min}$ \\
\hline $\boldsymbol{S}_{g, 0}$ & - & $\boldsymbol{k}_{c}$ & $\mathrm{ml}$ \\
\hline
\end{tabular}

Table 1: Parameters estimated by the volume kinetic model. In the first run, the glucose kinetics was solved and minimized (left columns). In the second run, the glucose parameters were used in the fluid kinetic run (right columns). ${ }^{*} / f$ present and identifiable.

\begin{tabular}{|c|c|c|c|c|c|}
\hline & \multirow{2}{*}{$\frac{k_{\mathrm{el}}}{\mathrm{ml} / \mathrm{min}(\S)}$} & \multirow{2}{*}{$\begin{array}{c}k_{b} \\
\operatorname{ml} / \min (\S)\end{array}$} & \multirow{2}{*}{$k_{c} \quad \mathrm{ml} / \mathrm{min}^{*} 10^{-3}(\S)$} & \multirow{2}{*}{$\begin{array}{l}V_{1} \\
m l\end{array}$} & \multirow{2}{*}{$T_{v}$} \\
\hline & & & & & \\
\hline PO-young & $30.2(18.8-54.3)^{\star}$ & $1.9(1.3-2.8)^{*}$ & $11.2(9.2-705.3)^{\star}$ & $947(375-2210)^{*}$ & $29.0(17.8-32.2)$ \\
\hline IV-young & $44.3(32.7-68.0)^{\star *}$ & $0.40(0.09-1.39)^{\star *}$ & $17.9(9.0-67.2)^{\star *}$ & $3560(2454-6375)^{\star *}$ & - \\
\hline PO-elderly & $49.2(32.6-115.8)^{*}$ & $0.74(0.15-1.62)^{*}$ & $34.1(15.7-80.8)^{\star}$ & $1233(275-2698)^{*}$ & $24.6(15.0-40.4)$ \\
\hline IV-elderly & $25.5(11.2-45.9)^{\star *}$ & $1.31(0.39-2.07)^{\star *}$ & $0.011 .8(0.4-15.8)^{* *}$ & $3027(2709-4553)^{\star *}$ & - \\
\hline
\end{tabular}

*No difference in lag time. ${ }^{*}$ No difference between administration routes but elderly had significantly larger half-time and larger deviations of plasma glucose levels from baseline.

Table 2: The estimates of the glucose kinetic parameters; $C L$ (clearance), $V_{d}$ (volume of distribution), $t_{1 / 2}$ (half-time) and $\mathrm{S}_{\mathrm{g}, 0} \%$ (a scalar indicating the shift from baseline of the plasma glucose concentration). § no significant difference between PO and IV experiments.

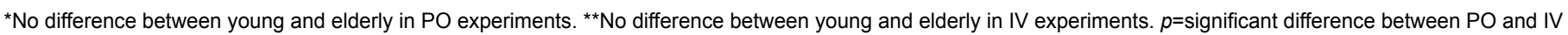
experiments $\left(V_{1}\right)$ while $\S$ presents no significant difference between $\mathrm{PO}$ and IV experiments $\left(k_{e l}\right.$ and $k_{b}$, young and elderly).

Table 3: The estimates of the fluid kinetic parameters. 

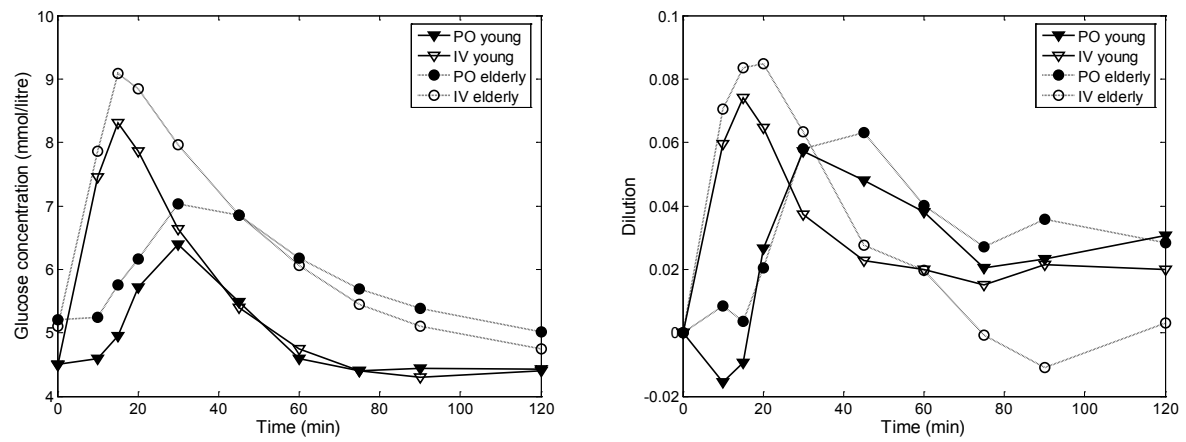

Figure 2:The mean glucose curves $(A)$ and the mean dilution $(B)$ for the four experiments.
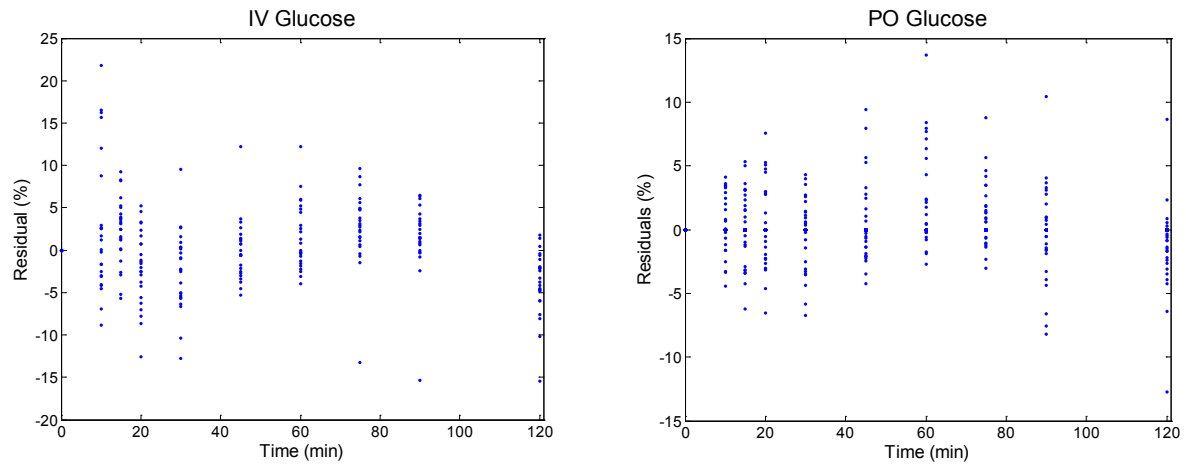

Figure 3: Residuals of the one-volume glucose kinetics.

\section{Glucose kinetics}

All subjects converged. In Figure 3, the relative residuals are shown.

No benefits could be seen by adding more compartments to the kinetic model for the glucose molecules (Table 3).

\section{Glucose kinetics-PO vs IV experiments, young versus old}

For both age groups, the plasma glucose concentration increase was more prominent when giving the glucose solution intravenously in comparison with orally. The maximum plasma glucose concentration and the continuous increase from baseline were higher for the elderly, regardless of administration route.

The $C L$ differed significantly both between administration routes ( $p=0.005$ PO highest) and age groups $\left(p<0.001\right.$ young highest). The $V_{d}$ differed between age groups ( $p=0.001$ elderly largest) but there was no significant difference between administration routes.

When selecting the PO experiments and analyzing any differences between the young and elderly subjects, the $C L$ was lower in the elderly ( $p=0.01)$ while the $V_{d}$ was larger in the elderly $(\mathrm{p}=0.002)$.

When selecting the IV experiments, no significant differences in $V_{d}$ could be found during the IV experiments while the elderly subjects had a lower $C L(p=0.002)$.

When selecting the young subjects, PO experiments gave a higher $C L(p=0.003)$ but there was no difference in $V_{d}$.

When selecting the elderly subjects, there were no significant differences in either $C L$ or $V_{d}$.
The lag time, $T_{o}$, did not differ between the age groups, i.e. there was no time difference in the first-pass metabolism.

\section{Bioavailability}

The bioavailability of glucose during PO experiments includes the assumption that glucose, and by osmosis also water molecules, reaches the plasma volume first after the glucose having gone through the first-pass metabolism in the liver. The bioavailability in this study was estimated to:

$$
\begin{aligned}
& F_{\text {elderly }}=0.65(0.60-0.69) \\
& F_{\text {young }}=0.70(0.59-0.94)
\end{aligned}
$$

This suggests that approximately $30 \%$ to $35 \%$ of the glucose does not reach the plasma when administrated orally. No significant difference could be seen in this regard between the age groups.

\section{Fluid kinetics}

Fluid kinetics-PO vs IV experiments, young versus old: The central volume, $V_{1}$, which is the theorized plasma volume in this kinetic model, differed between administration routes. When analyzing all PO and IV experiments, the plasma volume expanded significantly $(p<0.001)$ more when fluid was given intravenously. The larger expansion of the plasma volume during the IV experiments remained significant also when analyzing the subgroups young $(p=0.003)$ and elderly ( $p=0.005$ ) respectively. However, there was no difference seen in plasma volume expansion between age groups.

For the basic elimination rate $\left(k_{b}\right)$ and the elimination rate related to the plasma volume expansion $\left(k_{e}\right)$, no significant differences could 
be found between age groups or administration routes. The rate of fluid back to the plasma volume $(k)$ from the cellular volume $(V)$, was also at the same levels regardless of age and administration route. The lag time, $T_{v}$, did not differ between age groups.

\section{Discussion}

Our primary hypothesis was that there should be a lag-time in plasma volume expansion when comparing intravenous to oral administration of glucose containing fluid. The profiles of the dilution and the plasma glucose levels and the kinetic parameters $T_{0}$ and $T_{v}$ did show that there is indeed a lag-time when giving fluid orally compared to intravenously. The kinetic parameters did not differ between the age groups, but the maximal plasma volume expansion occurred 20-30 minutes later if given orally compared to intravenously. Quite unexpectedly though, already during the oral rehydration, the glucose $2.5 \%$ solution caused a decrease of the plasma volume compared to baseline in young individuals. Plasma volume reduction during intravenous administration of glucose solution has been shown in previous studies in young healthy subjects, but then approximately 30 $60 \mathrm{~min}$ after the intravenous infusion [8]. There are some differences between oral and intravenous experiments that are not completely clarified by this study's design that i.e. excluded sampling of insulin levels.

A secondary end-point was to compute the difference between age groups when looking at lag time and the maximum plasma volume expansion at 15 minutes $\left(\Delta v_{i}\right)$. Plasma volume expansion at 15 minutes was greater $(p=0.05)$ during IV than PO while the retention of fluid at the end of the experiments was positive with the exception of IV for the elderly that returned to the baseline plasma volume.

A possible explanation to the plasma volume decrease during oral rehydration might be correlated to the response of pulsatile release of insulin to pulsatile intake of glucose solutions (PO vs IV).

Glucose enters liver cells in the first-pass metabolism and the osmotic force drives water into the cells along with each molecule of glucose, and this occurs during the time the subject drinks the solution and 5-10 min after the drinking has ceased. This is underlined by the fact that the plasma glucose levels did not change in comparison to baseline during this timeperiod. When analyzing the $\mathrm{PO}$ administration route, an increase in both plasma glucose level and plasma volume expansion can be monitored when the hepatic glucose enzyme system has reached a high saturation level. In the elderly, both plasma glucose and plasma volume remained unchanged during the first $25 \mathrm{~min}$ of the oral experiment, probably as an effect of first-pass metabolism.

Clinically, the influx of water and glucose into the cells is many times a highly desired effect - to rehydrate the cells in people with a longstanding state of dehydration that many times is presented with symptoms such as dizziness, nausea, headache, and even a state of confusion

Previous studies on elderly have shown an almost $50 \%$ reduction in elimination of a glucose load which would indicate that relatively more glucose molecules pass the liver cells and cause a more rapidly increase in the plasma glucose levels [13]. Similarly, in diabetics and trauma patients, who also have a reduced elimination of glucose molecules, plasma volume expansion and increase in plasma glucose occur more rapidly when glucose solutions are given intravenously $[7,14]$.

The young had an almost doubled clearance (98\%) during the PO experiments while the $C L$ increased by $43 \%$ in the elderly.
Moreover, the young had a rather small $V_{d}(7.8 \mathrm{~L})$ compared to the elderly that presented a $V_{d}$ that was more than doubled the size of the young $(18.6 \mathrm{~L})$. The deviation from baseline of plasma glucose concentration, $S_{g} \%$, was smaller for the young subjects compared to the elderly. The effect of these results was an overall smaller increase of glucose concentration in plasma compared to the elderly.

From a kinetic point of view, the plasma glucose concentration depends on both the rate of the $C L$ and the size of the $V_{d}$. Having a fast $C L$ and a small $V_{d}$ might level out each other, and cause little effect on the glucose concentration in the plasma, i.e. there will be only small changes from the baseline. For the young subjects, these small effects were presented and the $S_{g} \%$ indicated, consequently, a smaller deviation from baseline compared to the elderly subjects.

We have not studied subjects with acute or chronic diseases, nor have we found previous publications comparing oral vs intravenous hydration in young and elderly. Therefore, our results and conclusions cannot be valid for clinical recommendations, but the results of this study indicate that oral rehydration takes longer time and is related to a transient reduction of plasma volume in younger individuals during which there are risks for unfavorable symptoms. In our small sample study, some of the young individuals complained having headache, dizziness and nausea during the first $60 \mathrm{~min}$ of the PO experiments.

Furthermore, the maximum plasma volume expansion is not as large when giving glucose-containing fluid orally compared to intravenously. However, it seems as though the attained level of plasma volume expansion sustains over a longer period of time when given orally to elderly. This might be due to a less potential influence of PO rehydration on hormone response causing a slower elimination of fluid administration.

The persistence in plasma volume expansion by oral rehydration supports the clinical rule of thumbs to give people fluid via oral, rather than via an intravenous route, for rehydration purposes. However, a common clinical problem is fluid overload of the interstitial compartments (oedema) that might question the oral route of rehydration if it is not influencing the hormone response in an effective way.

An important bed-side issue is the hard time especially elderly experience when have to drink even modest volumes of fluid. The participating subjects managed to drink this isotonic solution of fairly large quantities and doing so very fast. This is not a realistic treatment in a clinical setting with frail elderly patients. Therefore, this study indicates that intravenous rehydration must be considered when treating a dehydrated geriatric patient, especially at emergency departments where these patients quite often are first met and treatment first initiated. The dose of $7 \mathrm{ml} / \mathrm{kg}$ could well be adequate as a first bolus dose, which does not increase the plasma volume too much risking complications such as overload and pulmonary oedema.

The glucose kinetic parameters were generated by a onecompartment model that did not improve by adding more compartments. This finding is consistent with several other volume kinetic studies $[7,8,15]$.

\section{Conclusion}

By using a volume kinetic model, it was possible to calculate absorption, distribution and elimination of fluids given both orally and intravenously in two age groups, young and elderly. Compared to intravenous rehydration, about $1 / 3$ of the administered glucose 
Citation: Sjöstrand F, Svensen CH, Rodhe PM (2014) Oral Rehydration or Intravenous Drip?-A Volume Kinetic Study of Glucose Solutions to Young and Old. J Appl Computat Math 3: 190. doi:10.4172/2168-9679.1000190

never reached the plasma volume during oral rehydration which may explain the modest plasma volume expansion seen in both age groups. Young subject had a rapid efflux of water from the plasma volume at the initiation of oral rehydration causing a transient decrease of the plasma volume.

\section{Financial Support}

Stockholm County Grant.

\section{Acknowledgement}

We are very grateful to CEO Anna Biró, and to the staff, at Nackageriatriken AB. A special thanks to ward 51 and to Head Research Nurses Birgith Olsson and Gunilla Brohmé and Research Nurses Nana Waldréus, Helena Stenström and Johanna Pelttari.

\section{References}

1. Bennett JA, Thomas V, Riegel B (2004) Unrecognized chronic dehydration in older adults: examining prevalence rate and risk factors. J Gerontol Nurs 30 : 22-28.

2. Gordon JA, An LC, Hayward RA, Williams BC (1998) Initial emergency department diagnosis and return visits: risk versus perception. Ann Emerg Med 32: $569-573$.

3. Mentes JC, Culp K (2003) Reducing hydration-linked events in nursing home residents. Clin Nurs Res 12: 210-225.

4. Warren JL, Bacon WE, Harris T, McBean AM, Foley DJ, et al. (1994) The burden and outcomes associated with dehydration among US elderly, 1991. Am J Public Health 84: 1265-1269.
5. Mentes $\mathrm{J}$ (2006) Oral hydration in older adults: greater awareness is needed in preventing, recognizing, and treating dehydration. Am J Nurs 106: 40-49.

6. Sjöstrand F, Hahn RG (2003) Validation of volume kinetic analysis of glucose $2.5 \%$ solution given by intravenous infusion. $\mathrm{Br} \mathrm{J}$ Anaesth 90: 600-607.

7. Sjöstrand F, Nyström T, Hahn RG (2006) Intravenous hydration with a $2.5 \%$ glucose solution in Type II diabetes. Clin Sci (Lond) 111: 127-134.

8. Sjöstrand F, Berndtson D, Olsson J, Strandberg P, Hahn RG (2008) The osmotic link between hypoglycaemia and hypovolaemia. Scand J Clin Lab Invest 68: 117-122.

9. al MRDe. Miller's Anesthesia. Livingstone C, editor: Churchill Livingstone; 2009.

10. Sicardi Salomón Z, Rodhe P, Hahn RG (2006) Progressive decrease in glucose clearance during surgery. Acta Anaesthesiol Scand 50: 848-854.

11. Sjöstrand F, Edsberg L, Hahn RG (2001) Volume kinetics of glucose solutions given by intravenous infusion. $\mathrm{Br} \mathrm{J}$ Anaesth 87: 834-843.

12. Rodhe P, Drobin D, Hahn RG, Wennberg B, Lindahl C, et al. (2010) Modelling of peripheral fluid accumulation after a crystalloid bolus in female volunteers a mathematical study. Computational and mathematical methods in medicine 11: 341-351.

13. Miller R (2005) Anestesia: Churchill Livingstone.

14. Sjöstrand F, Hahn RG (2004) Volume kinetics of glucose $2.5 \%$ solution during laparoscopic cholecystectomy. Br J Anaesth 92: 485-492.

15. Hahn RG, Edsberg L, Sjöstrand F (2003) Volume kinetic analysis of fluid shifts accompanying intravenous infusions of glucose solution. Cell Biochem Biophys 39: 211-222. 